\title{
Filling carbon nanotubes with argon
}

\author{
G. S. Bocharov ${ }^{1}$, M. S. Egin $^{1}$, A. V. Eletskii ${ }^{1}$, V.L. Kuznetsov ${ }^{2}$ \\ ${ }^{1}$ National Research University MPEI, Moscow, Russia \\ ${ }^{2}$ G. K. Boreskov Institute of Catalysis, Sibirian Branch of RAS, Novosibirsk, Russia \\ eletskii@mail.ru
}

PACS 68.43.Mn, 68.43.Nr

DOI 10.17586/2220-8054-2018-9-1-85-88

The specific surface area of multi-walled carbon nanotubes (MWCNT) of different geometry and structures is measured by the method BET. The nanotubes were synthesized by the use of highly effective $\mathrm{Fe}-\mathrm{Co}$ catalysts through the method of polymerized complex precursors. In some cases, the measured specific surface area considerably exceeds that calculated under the assumption that the Ar adsorption occurs on the outer surface of CNTs. This permits one to conclude that in some cases a part of argon adsorbed fills the internal hollow of nanotubes.

Keywords: carbon nanotubes, specific surface area, sorption properties.

Received: 1 June 2017

Revised: 24 August 2017

\section{Introduction}

One of the prominent features of carbon nanotubes (CNT) relates to the existence of an internal cavity where various gaseous and condensed substances can be accumulated (see reviews [1-3] and the works cited there). This feature permits one to consider CNTs as a potential reservoir for storage of gases and harmful substances and to use them as a natural chamber for performing chemical reactions. Determination of the content of substances filling the internal hollow of CNTs requires complex apparatus, the usage of which is accompanied by an intense action onto the material and can result in the depletion of CNT cavity. Thus, the authors of the work [4] studied filling single walled CNT with nitrogen and oxygen using X-ray diffraction measurements and isotherm absorption analysis. It has been observed that molecular gases fill the inner cavity of CNTs only in the case of thermally treated samples resulting in opening the nanotube's ends. The adsorption capacity of single-wall carbon nanotubes (SWCNTs) bundles with regard to the pure $\mathrm{CH}_{4}, \mathrm{~N}_{2}, \mathrm{CO}$ and $\mathrm{CO}_{2}$ gases at $298 \mathrm{~K}$ and pressures ranging from 0.01 to $2.0 \mathrm{MPa}$ has been investigated experimentally and computationally in [5]. In the present work, special attention is paid to the possibility of relatively simple determination of gaseous argon content inside CNTs based on the analysis of the specific surface area measurements for the material.

\section{Experiment}

Multi-walled CNTs (MWCNT) of different geometry have been synthesized in G.K. Boreskov Institute of Catalysis, Sibirian Branch RAS, Novosibirsk, by the use of high effective Fe-Co catalysts through the method of polymerized complex precursors [6]. $\mathrm{Ca}, \mathrm{Al}$ and $\mathrm{Mg}$ ions were used as the catalyst precursors. In the classic version of this method, the salts of the selected metals are mixed with citric acid and ethylene glycol or ethylenediamine, the condensation of which at high temperature, results in the formation of a 3D polymer matrix. Practically homogeneous distribution of metal ions in the organic matrix is reached due to coordination of these ions with carboxyl groups of citric acid. Subsequent burning off the organic substance results in formation of a system of mixed spinel oxides. If this step is performed at relatively moderate temperatures, oxide particles obtained can be found in a dispersed, practically amorphous state [7-9]. Variation of the oxide component of catalyst $(\mathrm{Ca}, \mathrm{Al}$, $\mathrm{Mg}$ ), that is not reduced during the CNT synthesis and, in essence, a carrier, permits one to vary the dispersion of metal particles due to the reduction by the substrate (ethylene) [10]. This results in production of MWCNT having different distribution over diameter (MWCNT 1, MWCNT 2, MWCNT 3). One should note that processing these base samples (oxidation, grinding) did not result in change of their diameter distribution. Therefore these distributions are true also for functionalized samples.

The mean diameter of MWCNT was controlled through changing the concentration of the active component in the catalyst. Diameter distribution of MWCNT and structural changes of graphene shaving covering the nanotube's surface were determined by means of HR-TEM (JEM-2010). The diameter distribution was determined for each sample on the basis of analysis of 300-600 TEM images of MWCNTs (Fig. 1), obtained at magnification of $50,000 \times$ and $400,000 \times$. Fig. $2(a, b, c)$ present the typical diameter distributions for MWCNTs. These distributions 


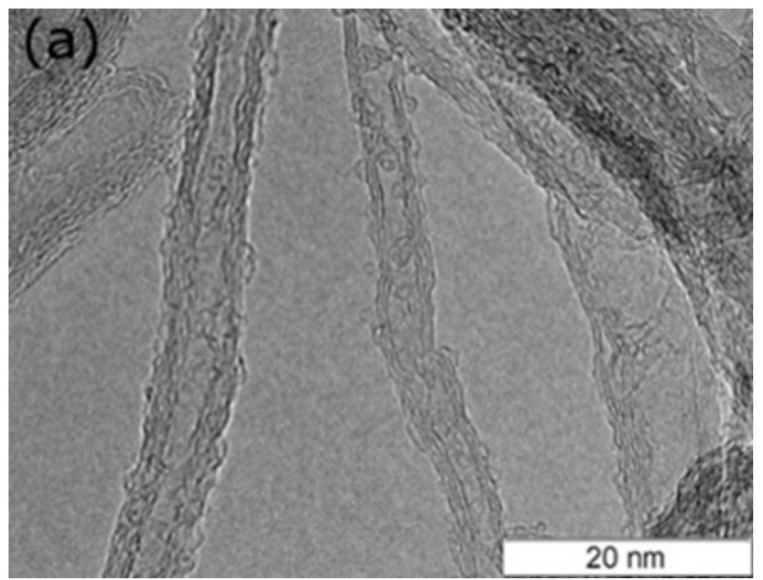

FIG. 1. CNT image of MWCNT-1

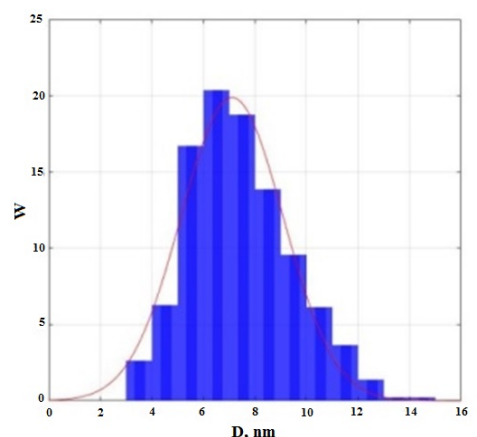

(a)

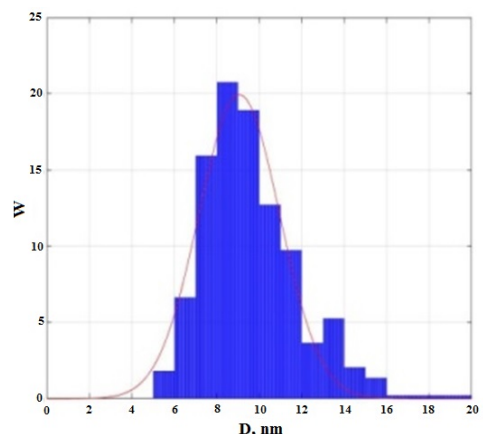

(b)

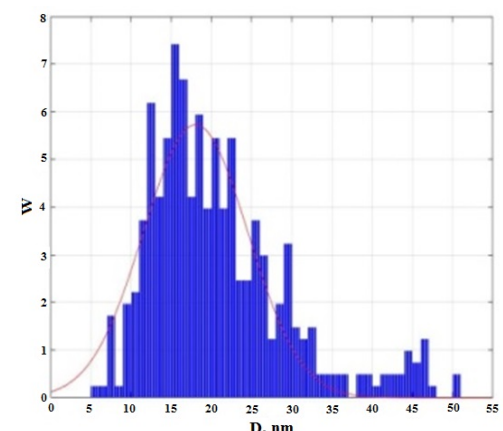

(c)

FIG. 2. Typical diameter distributions of MWCNT determined through the treatment of microimages: MWCNT-1 (a); MWCNT-2 (b); MWCNT-3 (c). The distributions have been normalized by unity. The diameter distributions smoothed by the Gauss function are shown by the lines

are readily reproducible for different sample sets produced with the use of specific catalyst types. The accuracy of determination of the maximum of the distribution accounts $\pm 0.5 \mathrm{~nm}$ for thin $(7-10 \mathrm{~nm})$ and $\pm 1.5 \mathrm{~nm}$ for thicker CNTs $(\sim 20 \mathrm{~nm})$. Characteristics of the multi-walled CNTs under investigation are given in Table 1.

\section{Specific surface area of MWCNTs}

The specific surface area of MWCNTs was measured by the standard BET method using the SORBI-M setup. MWCNT samples of 20-40 mg in mass were placed into a camera where the argon sorption-desorption cycles at a liquid nitrogen temperature were performed. The measured data were treated by means of a computer program and averaged by three cycles. The results of such a treatment are given in the $4^{\text {th }}$ column of Table 1 .

The calculation of the specific area of MWCNT samples was performed using the typical diameter distributions (Fig. 2(a,b,c)). Considering MWCNT as a solid cylinder of the density $\rho$ and $D$ in diameter, the external specific surface area $S$ of such a cylinder is expressed by the following relation:

$$
S=\frac{4}{(D \cdot \rho)} .
$$

This relation is valid if the nanotube length considerably exceeds its diameter, and the number of layers $n \gg 1$. If the nanotube is considered not as a solid medium but as a set of concentric cylinders inserted into each another and having the distance between the neighboring walls of $d=0.34 \mathrm{~nm}$, then the external specific surface area will depend on the radius of the smallest cylinder $r_{0}$ :

$$
S=\frac{D \sigma}{r_{0} n\left[1+\frac{(n-1) d}{r_{0}}\right]},
$$


TABLE 1. Characteristics of the samples under investigation

\begin{tabular}{|c|c|c|c|c|c|}
\hline \multirow{3}{*}{ Sample } & \multirow{3}{*}{$\begin{array}{c}\text { Mean } \\
\text { diameter(nm) }\end{array}$} & \multirow{3}{*}{$\begin{array}{l}\text { Mean length (the } \\
\text { width of } \\
\text { distribution) (nm) }\end{array}$} & \multicolumn{3}{|c|}{ Specific surface area $\left(\mathrm{m}^{2} / \mathrm{g}\right)$} \\
\hline & & & \multirow{2}{*}{ Measurement } & \multicolumn{2}{|c|}{ Calculation } \\
\hline & & & & (1) & (3) \\
\hline MWCNT-1-LRP ${ }^{1}$ & 7.2 & Up to 50000 & $342.2 \pm 1.9$ & 247 & 271 \\
\hline MWCNT-1-LRP APF ${ }^{2}$ & 7.2 & $220(20-500)$ & $285 \pm 12$ & 247 & 271 \\
\hline MWCNT-1-LRP APF $\mathrm{O}_{x} \mathrm{Na}^{3}$ & 7.2 & $220(20-500)$ & $323 \pm 15$ & 247 & 271 \\
\hline MWCNT-2-LRP ${ }^{1}$ & 9.4 & Up to 50000 & $320.0 \pm 1.9$ & 189 & 195 \\
\hline MWCNT-2-LRP APF & 9.4 & $220(20-600)$ & $290 \pm 3$ & 189 & 195 \\
\hline MWCNT-2-LRP APF $\mathrm{O}_{x} \mathrm{Na}^{3}$ & 9.4 & $220(20-600)$ & $328 \pm 5$ & 189 & 195 \\
\hline MWCNT-3-LRP $^{1}$ & 18 & Up to 30000 & 100.2 & 99 & 111 \\
\hline MWCNT-3-LRP APF ${ }^{2}$ & 18 & $520(20-500)$ & 113.2 & 99 & 111 \\
\hline MWCNT-3-LRP APF $\mathrm{O}_{x} \mathrm{Na}^{3}$ & 18 & $520(20-500)$ & 110.7 & 99 & 111 \\
\hline
\end{tabular}

${ }^{1}$ The catalyst is washed out. ${ }^{2}$ The catalyst is washed out, grinded. ${ }^{3}$ The catalyst is washed out, grinded, oxidized.

The carboxyl groups content $0.75-0.9 \mathrm{~nm}^{-1}$.

where $n \approx D / 2 d$ is the number of layers in the nanotube, $\sigma \approx 1300 \mathrm{~m}^{2} / \mathrm{g}$ is the specific surface area of the hexagonal plane constituting the basis of the nanotube structure. Using the obvious relation $\rho=(\sigma d)^{-1}$ the equation (2) is reduced to the more simple relation (1) under the condition $n \gg 1$. The Eq. (2) is more correct that (1), however it contains an indefinite parameter $r_{0}$ therefore the specific surface area was calculated using Eq. (1). The calculations imply that the error caused decreases as the outer nanotube' diameter $D$ increases and the smallest nanotube's radius $r_{0}$ decreases. The typical value of $r_{0}$ lies in the range of between 0.7 and $0.9 \mathrm{~nm}$, therefore for nanotubes with outer diameter of $7 \mathrm{~nm}$ this error accounts $8-10 \%$ while for more wide nanotubes $(D=9.4$ and $18 \mathrm{~nm})$ this error accounts $5-10$ and 2-4\%, correspondingly. Such an error is within the accuracy of determination of nanotube's diameter.

The external specific surface area is expressed as follows:

$$
S=\int_{0}^{\infty} \frac{4 W(D)}{(D \rho)} d D .
$$

Here, $W(D)$ is the diameter distribution of CNT (Fig. 2), which is approximated by the Gauss function. The results of calculation of the specific surface area of CNTs of different types are given in the 5-th and 6-th columns of Table 1. The data presented in the 5-th column have been obtained on the basis of Eq. (1) supposing that all the nanotubes have a fixed (mean) diameter. The data of the 6-th column have been obtained on the basis of Eq. (3) with taking into account the real CNT diameter distribution. As is seen, taking into consideration the real CNT diameter distribution changes the calculated values of the specific surface area within the range of $10 \%$.

The analysis of the specific surface area values presented in Table 1 indicates that for samples MWCNT1 and MWCNT2 the measured values of the specific surface area exceed by $20-30 \%$ those obtained through the calculation of the external specific surface area. This permits one to conclude that the nanotubes of the abovementioned types not only adsorb argon by their external surface bit also store it in its inner cage.

\section{Conclusion}

The specific surface area of MWCNT produced by using highly productive Fe-Co based catalysts utilizing the method of polymerized complex precursors has been measured by the BET method. Argon at a liquid nitrogen temperature has been used as an adsorbed gas. For some MWCNT samples, the measured value of the specific surface area notably exceeded the relevant calculation data obtained under the assumption that argon is desorbed 
by the external surface of the nanotubes. Therefore, argon is adsorbed by both the external surfaces and by the internal cavities of the nanotubes under consideration.

\section{Acknowledgements}

The work is supported by the State Tasks No 3.1414.2017 and No 3.7131.2017.

\section{References}

[1] Eletskii A.V. Sorption properties of carbon nanostructures. Physics-Uspechi, 2004, 47(11), P. 1119-1154.

[2] Zhao J. Gas Adsorption of Carbon Nanotubes: Tube-Molecule Interaction and Technological Applications. Current Nanoscience, 2005, 1(2), P. 169-176.

[3] Pietraß T. Gas Adsorption on Carbon Nanotubes. In: Modern Magnetic Resonance, ed. by G. A. Webb. Springer, 2008, P. 1479-1485.

[4] Fujiwara A., Ishii K., Suematsu H., Kataura H., Maniwa Y., Suzuki S., Achiba Y. Gas adsorption in the inside and outside of single-walled carbon nanotubes. Chem. Phys. Lett., 2001, 336, P. 205-211.

[5] Lithoxoos G.P., Labropoulos A., Peristeras L.D., Kanellopoulos N., Samios J., Economou I.G. Adsorption of $\mathrm{N}_{2}$, $\mathrm{CH}_{4}$, $\mathrm{CO}_{\text {and }} \mathrm{CO}_{2}$ Gases in Single Walled Carbon Nanotubes: A Combined Experimental and Monte Carlo Molecular Simulation Study. J. Supercrit. Fluids, 2010, 55(2), P. 510-523.

[6] Kakihana M., Yoshimura M. Synthesis and Characteristics of Complex Multicomponent Oxides Prepared by Polymer Complex Method. Bull. Chem. Soc. Japan, 1999, 72(7), P. 1427-1443.

[7] Usoltseva A., Kuznetsov V., Rudina N., Moroz E., Haluska M., Roth S. Influence of catalysts' activation on their activity and selectivity in carbon nanotubes synthesis. Phys. Status Solidi , 2007, 244(11), P. 3920-3924.

[8] Kuznetsov V.L., Usoltseva A.N. Method of production fine-grained supported catalysts and synthesis of carbon nanotubes. Patent.2373995 Russia: 27.11.2009 Bull. 33.

[9] Elumeeva K.V. Production of multi-walled carbon nanotubes with predetermined properties. Thesis. Novosibirsk. Institute of Catalysis, Siberian Branch of RAS, 2012, $161 \mathrm{p}$

[10] Kuznetsov V.L., Krasnikov D.V., Schmakov A.N., Elumeeva K.V. In situ and ex situ time resolved study of multi-component Fe-Co oxide catalyst activation during MWNT synthesis. Phys. Status Solidi B, 2012, 249(12), P. 2390-2394. 